\title{
Absolute Lymphocyte/Monocyte Ratio at Diagnosis and Interim Positron-Emission Tomography Predict Survival in Classical Hodgkin Lymphoma
}

\author{
Luis F. Porrata", Kay M. Ristow, Thomas M. Habermann, Thomas E. Witzig, Joseph P. Colgan, \\ David J. Inwards, Stephen M. Ansell, Ivana N. Micallef, Patrick B. Johnston, Grzegorz Nowakowski, \\ Carrie A.Thompson, Svetomir N. Markovic
}

Division of Hematology, Internal Medicine, Mayo College of Medicine, Rochester, USA.

Email: " porrata.luis@mayo.edu

Received January $15^{\text {th }}, 2013$; revised February $17^{\text {th }}, 2013$; accepted February $26^{\text {th }}, 2013$

\begin{abstract}
Interim Positron-Emission Tomography (int-PET) and the peripheral blood absolute lymphocyte/monocyte ratio at diagnosis (ALC/AMC-DX) have been shown to be predictors for progression-free survival (PFS) and time to progression (TTP) in classical Hodgkin lymphoma (cHL). Therefore, we studied if the combination of ALC/AMC-DX and the (int-PET) can further stratified PFS and TTP in cHL patients. Patients were required to be diagnosed, treated, and followed with int-PET at Mayo Clinic, Rochester, Minnesota. From 2000 until 2008, $111 \mathrm{cHL}$ patients qualified for the study. The median follow-up was 2.8 years (range: $0.3-10.4$ years). Patients with a negative int-PET $(\mathrm{N}=98)$ presented with a higher ALC/AMC-DX (median of 2.32, range: 0.26 - 37.5) compared with patients with a positive int-PET $(\mathrm{N}=13)$ (median of 0.9 , range: $0.29-3.10), \mathrm{p}<0.004$. By multivariate analysis, ALC/AMC-DX and the int-PET were independent predictors for PFS and TTP, when compared with the International prognostic Score. Patients were stratified into four groups: group 1 included patients with a negative int-PET and ALC/AMC-DX $\geq 1.1$; group 2 included positive int-PET and ALC/AMC-DX $\geq 1.1$; group 3 included negative int-PET and ALC/AMC-DX $<1.1$; and group 4 included positive int-PET and ALC/AMC-DX $<1.1$. Group 1 experienced superior PFS and TTP in comparison with the other groups. In conclusion, the combination of ALC/AMC-DX and the int-PET provides a simple model to assess clinical outcomes in cHL.
\end{abstract}

Keywords: Classical Hodgkin Lymphoma; Absolute Lymphocyte/Monocyte Ratio at Diagnosis; Interim PET-Scan; Progression-Free Survival; Time to Progression

\section{Introduction}

The peripheral blood absolute lymphocyte/monocyte ratio at diagnosis (ALC/AMC-DX), as a surrogate biomarker of host immunity (i.e., ALC) and tumor microenvironment (i.e., AMC), has been recently reported to be a predictor for overall survival, lymphoma-specific survival, progression-free survival, and time to progression in classical Hodgkin lymphoma (cHL) [1,2]. Interim PositronEmission Tomography (int-PET), as a functional imaging test for tumor activity, has been shown to be a predictor for progression-free survival (PFS) and time to progression (TTP) in cHL [3]. Romano et al. [4] reported that even though the ALC/AMC ratio correlated with prognosis, the int-PET was better discriminator of survival than the ALC/AMC ratio.

Therefore, in an attempt to validate Romano's findings,

"Corresponding author. we studied if the ALC/AMC-DX is an independent predictor in comparison with the int-PET for PFS and TTP and if the combination of ALC/AMC-DX and the intPET can further stratified the clinical outcomes of PFS and TTP in patients with cHL.

\section{Patients and Methods}

\subsection{Patient Population}

In order to participate in the study patients were required to have newly diagnosed cHL, treated with adriamycin, bleomycin, vinblastine, and dacarbazine (ABVD) with or without radiation, had an int-PET before cycle number 3, and be followed at the Mayo Clinic, Rochester, Minnesota.

Patients diagnosed with nodular lymphocyte predominant Hodgkin's lymphoma, treated only with radiation or palliative care, positive for human immunodeficiency 
virus and with concomitant autoimmune disease receiving immunosuppressive therapy were excluded. From 2000 to 2008, 111 consecutive cHL qualified for the study. No patients refused authorization to use their medical records for research and none was lost to follow-up. Approval for the retrospective review of these patients' records was obtained from the Mayo Clinic Institutional Review Board and the research was conducted in accordance with the USA federal regulations and the Declaration of Helsinki.

\subsection{End-Point}

The primary end-point of the study was to assess if the combination of ALC/AMC-DX and the int-PET can further stratified the clinical outcomes of PFS and TTP in patients with cHL.

The cut-off for the ALC/AMC-DX $\geq 1.1$ used in this study was based on our previous publication [1] and obtained from the complete blood cell count (CBC) at diagnosis of cHL. The ALC/AMC ratio was obtained by dividing the absolute lymphocyte count (ALC) over the absolute monocyte count (AMC) from the $\mathrm{CBC}$ at diagnosis [5].

\subsection{Prognostic Factors}

The prognostic factors evaluated in the study included the International Prognostic Score (IPS) [6] at diagnosis for advanced stage patients : [age $>45$ years, albumin $<$ $4 \mathrm{~g} / \mathrm{dl}$, ALC $<600 / \mu \mathrm{l}$ or $<8 \%$ of white cell count, hemoglobin $<10.5 \mathrm{~g} / \mathrm{dl}$, male gender, stage IV, and white blood cell count $\geq 15,000 / \mu \mathrm{l}]$; treatment modality (combination chemotherapy plus radiation versus chemotherapy alone), limited versus advanced disease, AMC at diagnosis, ALC/AMC ratio at diagnosis, and interim PETscan.

\subsection{Response and Survival}

Definitions of response criteria, PFS, and TTP were based on the guidelines from the International Harmonization Project Lymphoma [7]. PFS was defined as the time from $\mathrm{cHL}$ diagnosis to the time to progression, relapse from complete response, death as a result of any cause, or last follow-up. TTP was defined as the time from cHL diagnosis to the time of lymphoma progression, death as a result of lymphoma, or last follow-up.

\subsection{Statistical Analysis}

PFS and TTP were analyzed using the approach of Kaplan and Meier [8]. Differences between survival curves were tested for statistical significance using the twotailed log-rank test. The Cox proportional hazard model
[9] was used for the univariate and multivariate analyses to evaluate the variables under the prognostic factors' section to assess their impact on PFS and TTP. Chisquare tests were used to determine relationships between categorical variables. The Wilcoxon-rank test was used to determine associations between continuous variables and categories, and Spearman's correlation coefficients were used to evaluate associations for continuous variables. All $p$ values are two-sided associations and $p$ values less than 0.5 are considered statistically significant.

\section{Results}

\subsection{Patients Characteristics}

The median age at diagnosis for this cohort of $111 \mathrm{cHL}$ patients was 37 years (range: 18 - 83 years). The distribution of additional baseline characteristics for these patients is presented in Table 1. The median follow-up period for the cohort was 2.8 years (range: $0.3-10.4$ years) and for living patients $(\mathrm{N}=99)$ was 3.0 years (range: 0.3 - 10.4 years). Six patients died of causes not related to lymphoma or the treatment of lymphoma, and 6 patients did secondary to relapse/progression of lymphoma.

\subsection{ALC/AMC-DX, Int-PET, and Progression-Free Survival and Time to Progression}

Kaplan-Meier analysis was used to study the PFS and TTP based on the ALC/AMC-DX and int-PET. Patients with an ALC/AMC-DX $\geq 1.1$ experienced superior PFS (Figure 1(a)) and TTP (Figure 1(b)) compared with patients with an ALC/AMC-DX < 1.1 [PFS: median was not reached vs 8.6 years, 3 -year PFS rates of $92 \%(95 \%$ CI, $83 \%-99 \%)$ vs $50 \%(95 \%$ CI, $18 \%-80 \%)$, p < 0.0001, respectively; and TTP: median was not reached vs not reached, 3-year TTP rates of $96 \%$ (95\% CI, $87 \%$ $100 \%)$ vs $55 \%(95 \%$ CI, $20 \%-77 \%)$, p $<0.0001$, respectively]. Similarly, patients with a negative int-PET also experienced superior PFS (Figure 1(c)) and TTP (Figure 1(d)) compared with patients with a positive intPET [PFS: median was not reached vs 2.1 years, 3 -year PFS rates of $91 \%(95 \%$ CI, $85 \%$ - 98\%) vs $45 \%$ (95\% CI, $10 \%-80 \%), \mathrm{p}<0.0001$, respectively; and TTP: median was not reached vs 2.1 years, 3 -year TTP rates of $98 \%$ $(95 \%$ CI, $84 \%-100 \%)$ vs $45 \%$ (95\% CI, $10 \%-82 \%)$, p $<0.0001$, respectively].

By univariate analysis (Table 2), both ALC/AMC-DX and int-PET were predictors for PFS and TTP. Due to the strong correlation between ALC $(r=0.5, \mathrm{p}<0.0001)$ and AMC $(r=-0.4, \mathrm{p}<0.0001)$, we only included the ALC/AMC-DX in the multivariate analysis to prevent co-linearity. Similarly, both age and stage are included in 
Table 1. Baseline patients' characteristics at diagnosis.

\begin{tabular}{|c|c|c|c|}
\hline Variables & $\mathbf{N}(111)$ & Median & Range \\
\hline Age, years, median (range) & $111(100 \%)$ & 37 & $(18-83)$ \\
\hline \multicolumn{4}{|l|}{ Gender } \\
\hline Female & $51(46 \%)$ & & \\
\hline Male & $60(54 \%)$ & & \\
\hline Absolute lymphocyte count at diagnosis $\times 10^{9} / 1$, median (range) & $111(100 \%)$ & 1.38 & $(0.15-9.1)$ \\
\hline Albumin (g/dl), median (range) & $96(86 \%)$ & 4.0 & $(2.1-5.8)$ \\
\hline Hemoglobin (g/dl), median (range) & $111(100 \%)$ & 13.1 & $(9.0-17.2)$ \\
\hline Absolute monocyte count at diagnosis $\times 10^{9} / 1$ & $111(100 \%)$ & 0.61 & $(0.21-2.61)$ \\
\hline \multicolumn{4}{|l|}{ Stage } \\
\hline Limited & $56(50 \%)$ & & \\
\hline Advanced & $55(50 \%)$ & & \\
\hline $\mathrm{WBC} \times 10^{9} / 1$ & $111(100 \%)$ & 8.5 & $(1.8-21.0)$ \\
\hline \multicolumn{4}{|l|}{ Bulky disease } \\
\hline$\geq 10 \mathrm{~cm}$ & $2(25)$ & & \\
\hline$<10 \mathrm{~cm}$ & $109(98 \%)$ & & \\
\hline \multicolumn{4}{|l|}{ Treatment } \\
\hline Chemotherapy & $63(57 \%)$ & & \\
\hline Chemotherapy + radiation & $48(43 \%)$ & & \\
\hline \multicolumn{4}{|l|}{ IPS risk factors } \\
\hline \multicolumn{4}{|l|}{ Age in years } \\
\hline$>45$ & $39(35 \%)$ & & \\
\hline$\leq 45$ & $72(65 \%)$ & & \\
\hline \multicolumn{4}{|l|}{$\operatorname{Albumin}(\mathrm{g} / \mathrm{dl})(\mathrm{N}=96)$} \\
\hline$\geq 4$ & $51(53 \%)$ & & \\
\hline$<4$ & $45(47 \%)$ & & \\
\hline \multicolumn{4}{|l|}{ Absolute lymphocyte count per $\mu 1$} \\
\hline$\geq 600$ & $100(90 \%)$ & & \\
\hline$<600$ & $11(10 \%)$ & & \\
\hline \multicolumn{4}{|l|}{ Hemoglobin $(g / d l)$} \\
\hline$>10.5$ & $98(88 \%)$ & & \\
\hline$\leq 10.5$ & $13(12 \%)$ & & \\
\hline \multicolumn{4}{|l|}{$\mathrm{WBC} \times 10^{9} / 1$} \\
\hline$>15$ & $11(10 \%)$ & & \\
\hline$\leq 15$ & $100(90 \%)$ & & \\
\hline \multicolumn{4}{|l|}{ Stage 4} \\
\hline Yes & $23(21 \%)$ & & \\
\hline No & $88(79 \%)$ & & \\
\hline \multicolumn{4}{|l|}{ Number of IPS risk factors } \\
\hline 0 & $13(12 \%)$ & & \\
\hline 1 & $35(32 \%)$ & & \\
\hline 2 & $35(32 \%)$ & & \\
\hline 3 & $16(14 \%)$ & & \\
\hline 4 & $9(8 \%)$ & & \\
\hline 5 & $3(2 \%)$ & & \\
\hline \multicolumn{4}{|l|}{ IPS factors index } \\
\hline$\geq 3$ & $28(25 \%)$ & & \\
\hline$<3$ & $83(75 \%)$ & & \\
\hline \multicolumn{4}{|l|}{ PET-scan } \\
\hline Positive & $13(12 \%)$ & & \\
\hline Negative & $98(88 \%)$ & & \\
\hline
\end{tabular}

Abbreviations: IPS = International Prognostic Score; PET = Positron Emission Tomography; and WBC = white blood cell count. 


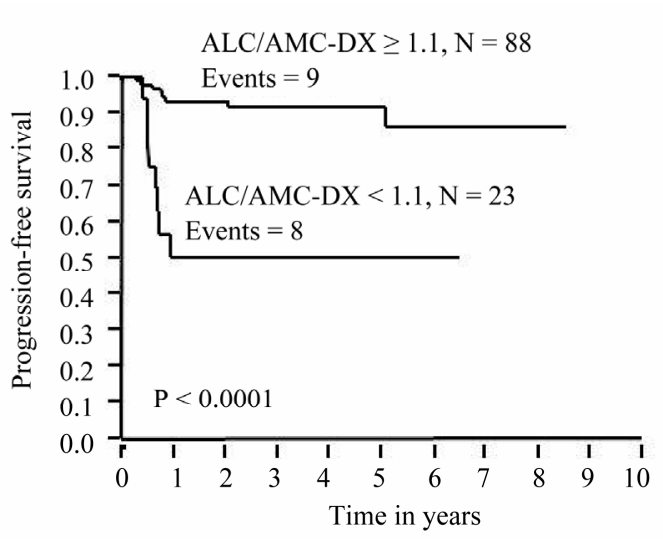

(a)

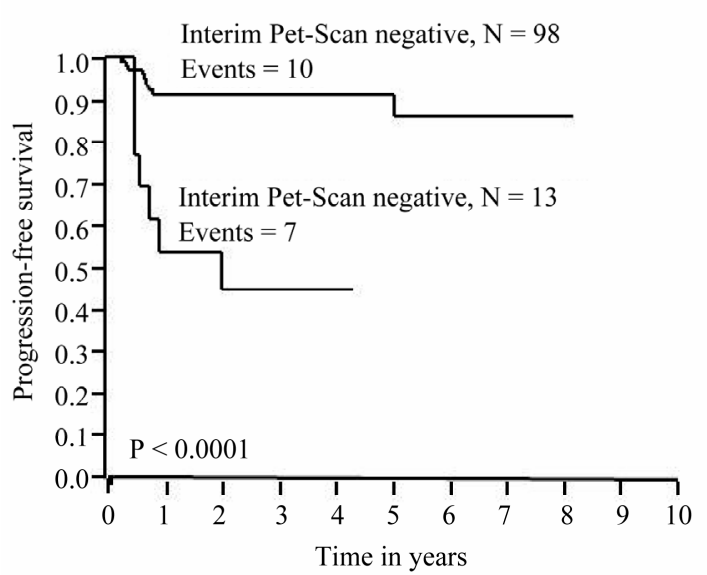

(c)

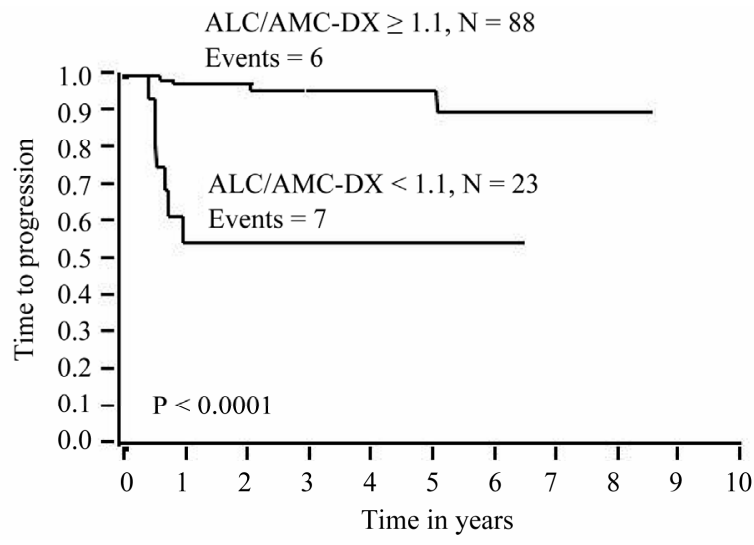

(b)

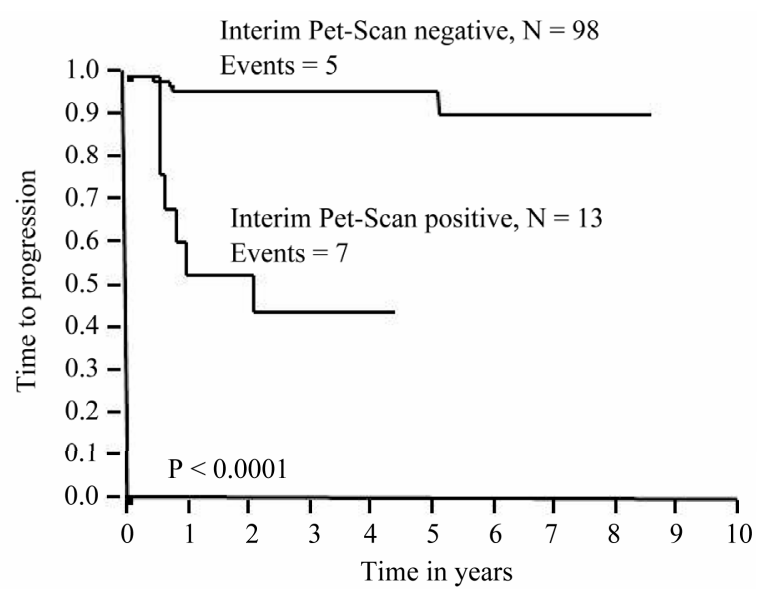

(d)

Figure 1. (a) Progression-free survival based on absolute lymphocyte/monocyte count ratio at diagnosis (ALC/AMC-DX); (b) Time to progression based on the ALC/AMC-DX; (c) Progression-free survival based on the interim positron emission tomography (PET)-scan; (d) Time to progression based on the interim PET-scan.

Table 2. Univariate analysis for progression-free survival and time to progression.

\begin{tabular}{|c|c|c|c|c|c|c|}
\hline \multirow[t]{2}{*}{ Variables } & \multicolumn{3}{|c|}{ Progression-free survival } & \multicolumn{3}{|c|}{ Time to Progression } \\
\hline & HR & $95 \% \mathrm{CI}$ & $\mathrm{p}$ & HR & $95 \% \mathrm{CI}$ & $\mathrm{p}$ \\
\hline Age $>45$ years & 3.52 & $1.30-10.35$ & $<0.01$ & 1.81 & $0.52-6.02$ & 0.3 \\
\hline Albumin $\geq 4 \mathrm{~g} / \mathrm{dl}$ & 0.35 & $0.10-1.06$ & 0.06 & 0.26 & $0.04-1.06$ & 0.06 \\
\hline $\mathrm{ALC} \geq 600 \mathrm{cel} 1 / \mu \mathrm{l}$ & 0.27 & $0.09-0.97$ & $<0.04$ & 0.15 & $0.04-0.58$ & $<0.009$ \\
\hline $\mathrm{AMC} \geq 630$ cells $/ \mu \mathrm{l}$ & 3.42 & $1.72-9.22$ & $<0.02$ & 3.70 & $1.06-12.31$ & $<0.04$ \\
\hline ALC/AMC-DX $\geq 1.1$ & 0.13 & $0.05-0.35$ & $<0.0002$ & 0.07 & $0.02-0.24$ & $<0.0001$ \\
\hline Bulky disease $\geq 10 \mathrm{~cm}$ & 3.74 & $0.21-18.76$ & 0.3 & 6.08 & $0.33-33.10$ & 0.2 \\
\hline $\mathrm{Hgb}<10.5 \mathrm{~g} / \mathrm{dl}$ & 1.04 & $0.16-3.71$ & 0.9 & 1.56 & $0.24-6.07$ & 0.6 \\
\hline IPS Factors $\geq 3$ & 4.14 & $1.54-11.60$ & $<0.005$ & 8.51 & $2.46-38.88$ & $<0.0006$ \\
\hline Limited disease & 0.12 & $0.02-0.44$ & $<0.0005$ & 0.19 & $0.13-0.73$ & $<0.01$ \\
\hline Male & 1.55 & $0.58-4.55$ & 0.4 & 2.50 & $0.72-11.42$ & 0.2 \\
\hline PET-scan negative & 0.13 & $0.05-0.37$ & $<0.0003$ & 0.05 & $0.01-0.18$ & $<0.0001$ \\
\hline $\mathrm{CT}+\mathrm{RT}$ vs $\mathrm{CT}$ alone & 0.08 & $0.004-0.38$ & $<0.0004$ & 0.11 & $0.01-0.60$ & $<0.007$ \\
\hline Stage 4 & 2.40 & $0.81-6.48$ & 0.1 & 2.35 & $0.61-7.83$ & 0.2 \\
\hline $\mathrm{WBC} \geq 15$ cells $/ \mu 1$ & 1.65 & $0.33-29.80$ & 0.6 & 1.04 & $0.21-20.26$ & 0.9 \\
\hline
\end{tabular}

Abbreviations: $\mathrm{ALC}=$ absolute lymphocyte count; $\mathrm{AMC}=$ absolute monocyte count $\mathrm{CT}=$ chemotherapy; $\mathrm{DX}=$ diagnosis; Hgb $=$ hemoglobin; IPS $=$ International Prognostic Score; PET = Positron Emission Tomography; RT = radiation; and WBC = white blood cell count. 
the IPS, we only included IPS in the multivariate analysis. In a multivariate Cox regression model (Table 3), both ALC/AMC-DX and int-PET were the only two statistically significant independent predictors for PFS and TTP. Since ALC/AMC-DX and int-PET were the only independent predictors in the initial multivariate analysis, we compared them in a subsequent multivariate analysis adjusting for each of them separately. Both remained still independent predictors for PFS [ALC/AMC-DX: HR = $0.51,95 \% \mathrm{CI}, 0.27-0.85, \mathrm{p}<0.005$ and int-PET: $\mathrm{HR}=$ $0.24,95 \% \mathrm{CI}, 0.07-0.76, \mathrm{p}<0.02]$ and for TTP $[\mathrm{ALC} /$ AMC-DX: HR $=0.42,95 \%$ CI, $0.17-0.83, p<0.006$ and int-PET: $\mathrm{HR}=0.11,95 \% \mathrm{CI}, 0.02-0.44, \mathrm{p}<0.002]$.

\subsection{Association between ALC/AMC-DX and Int-PET}

Because in this cohort of patients, only the ALC/AMCDX and int-PET were the only independent predictors for PFS and TTP, we set out to investigate if there was any association between the ALC/ACM-DX and int-PET. By the Wilcoxon-rant test, patients with a negative int-PET $(\mathrm{n}=98)$ presented with higher ALC/ AMC-DX (median of 2.32, range: 0.26 - 37.5) compared with patients with a positive int-PET $(\mathrm{n}=13)$ (median of 0.9 , range: 0.29 $3.10), p<0.004$ (Figure 2). Ninety percent (88/98) of patients with an ALC/AMC-DX $\geq 1.1$ achieved a negative int-PET compared to only $10 \%(10 / 98)$ of patients with an ALC/AMC-DX $<1.1, \mathrm{p}<0.0006$.

\subsection{ALC/AMC-DX and Int-PET Stratified Groups}

Because both the ALC/AMC-DX and int-PET were independent predictors for PFS and TTP, patients were stratified into four groups: group 1 included patients with a negative int-PET and ALC/AMC-DX $\geq 1.1$; group 2 included positive int-PET and ALC/AMC-DX $\geq 1.1$; group 3 included negative int-PET and ALC/AMC-DX < 1.1; and group 4 included positive int-PET and ALC/ AMC-DX $<1.1$. The three year PFS rates (Figure 3(a)) for each group were: $95 \%$ (group 1); $45 \%$ (group 2); $56 \%$ (group 3); and 50\% (group 4), $\mathrm{p}<0.0001$. The three year TTP rates (Figure 3(b)) for each group were: 100\% (group 1); $40 \%$ (group 2); 65\% (group 3); and 50\% (group 4), $\mathrm{p}<0.0001$.

\subsection{PFS and TTP Based on ALC/AMC-DX and Int-PET by Limited/Advanced Stage}

Because the IPS is only limited to advance stage cHL, we analyzed if both the ALC/AMC-DX and int-PET are predictors for PFS and TTP based on limited/advanced stage. Table 4 summarizes the univariate analysis for PFS and TTP based on ALC/AMC-DX and int-PET according to the sub-groups of limited stage and advanced stage. The ALC/AMC-DX and int-PET were predictors for PFS and TTP regardless of limited stage or advanced stage. We also compared group 1 (negative int-PET and ALC/AMC-

Table 3. Multivariate analysis progression-free survival and time to progression.

\begin{tabular}{cccccccc}
\hline Variables & \multicolumn{3}{c}{ Progression-free survival } & \multicolumn{3}{c}{ Time to progression } \\
\hline ALC/AMC-DX $\geq 1.1$ & 0.63 & $0.33-0.90$ & $<0.04$ & 0.16 & $0.03-0.63$ & $<0.004$ \\
IPS Factors $\geq 3$ & 2.47 & $0.83-7.60$ & 0.1 & 1.72 & $0.58-8.80$ & 0.5 \\
PET-scan negative & 0.22 & $0.07-0.71$ & $<0.02$ & 0.07 & $0.01-0.31$ & $<0.0004$ \\
CT + RT vs CT alone & 0.22 & $0.01-2.89$ & 0.4 & 0.17 & $0.01-2.79$ & 0.2 \\
\hline
\end{tabular}

Abbreviations: $\mathrm{ALC}=$ absolute lymphocyte count; $\mathrm{AMC}=$ absolute monocyte count; $\mathrm{CT}=$ chemotherapy; $\mathrm{DX}=$ diagnosis; IPS $=$ International Prognostic Score; PET = Positron Emission Tomography; and RT = radiation.

Table 4. Univariate analysis based on ALC/AMC-DX and int-PET according to limited stage and advanced stage.

\begin{tabular}{|c|c|c|c|c|c|c|}
\hline & \multicolumn{3}{|c|}{ Limited stage } & \multicolumn{3}{|c|}{ Advanced stage } \\
\hline \multicolumn{7}{|l|}{ Progression-free survival } \\
\hline ALC/AMC-DX $\geq 1.1$ & 0.07 & $0.01-0.21$ & $<0.0007$ & 0.58 & $0.33-0.95$ & $<0.02$ \\
\hline Int-PET negative & 0.10 & $0.02-0.20$ & $<0.002$ & 0.26 & $0.09-0.82$ & $<0.03$ \\
\hline Group 1 versus other & 0.04 & $0.01-0.18$ & $<0.002$ & 0.26 & $0.08-0.76$ & $<0.01$ \\
\hline \multicolumn{7}{|l|}{ Time to progression } \\
\hline ALC/AMC-DX $\geq 1.1$ & 0.06 & $0.01-0.22$ & $<0.0007$ & 0.41 & $0.11-0.82$ & $<0.007$ \\
\hline Int-PET negative & 0.11 & $0.03-0.21$ & $<0.002$ & 0.10 & $0.20-0.30$ & $<0.002$ \\
\hline Group 1 versus other & 0.05 & $0.02-0.17$ & $<0.002$ & 0.05 & $0.01-0.30$ & $<0.0003$ \\
\hline
\end{tabular}

Abbreviations: $\mathrm{ALC}=$ absolute lymphocyte count; $\mathrm{AMC}=$ absolute monocyte count; $\mathrm{DX}=$ diagnosis; group $1=$ negative int-PET and ALC/AMC-DX $\geq 1.1$; and PET $=$ Positron Emission Tomography. 


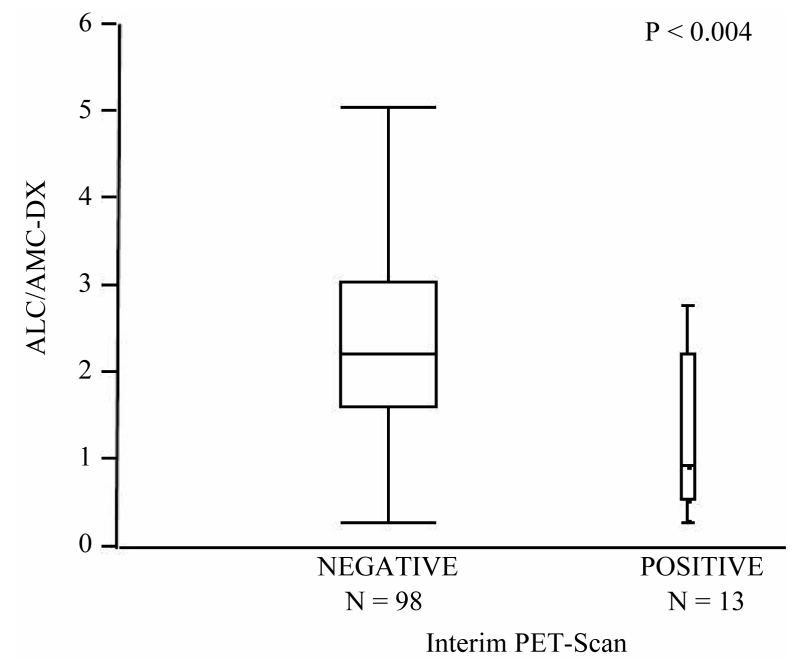

Figure 2. Box plot showing patients with a negative interim positron emission tomography (PET)-scan presented with higher absolute lymphocyte/monocyte count at diagnosis (ALC/AMC-DX) (median of 2.32, range: 0.26 - 37.5) compared with patients with a positive interim PET-scan (median of 0.9 , range: $0.29-3.10$ ).

$\mathrm{DX} \geq 1.1)$ versus other stratified groups described in the above 3.4 sub-heading. The stratified group 1 was also a predictor for PFS and TTP regardless of limited or advanced stage. Due to the small numbers of events by each sub-group, multivariate analysis was not performed.

\section{Discussion}

The int-PET representing a functional imaging test for tumor burden activity and the ALC/AMC-DX representing a surrogate biomarker for the interaction between host immunity and tumor microenvironment have been reported to predict clinical outcomes, specifically PFS and TTP in cHL. We, therefore, combined the int-PET and the ALC/AMC-DX as representative markers of the interaction between tumor burden, host immunity, and tumor microenvironment to further stratified clinical outcomes in cHL.

To support the hypothesis that the combination of the int-PET and the ALC/AMC-DX can further stratified PFS and TTP in CHL patients, it was necessary to demonstrate that both int-PET and the ALC/AMC-DX were independent predictors of PFS and TTP in cHL. In our cohort, by univariate analysis, both the int-PET and the ALC/AMC-DX were predictors for PFS and TTP. By multivariate analysis, both the int-PET and the ALC/ AMC-DX remained independent predictors for PFS and TTP when compared with the International Prognostic Score, limited or advanced disease, and chemotherapy with or without radiation. Because the int-PET and the ALC/AMC-DX in cohort of patients were the only two

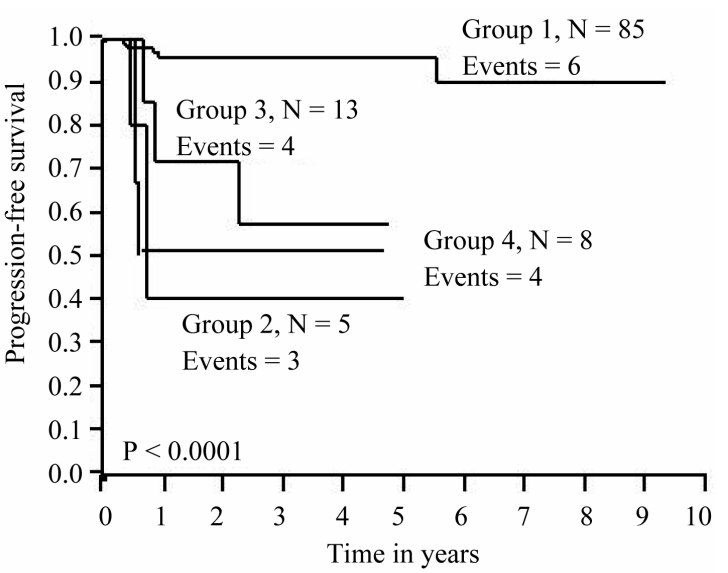

(a)

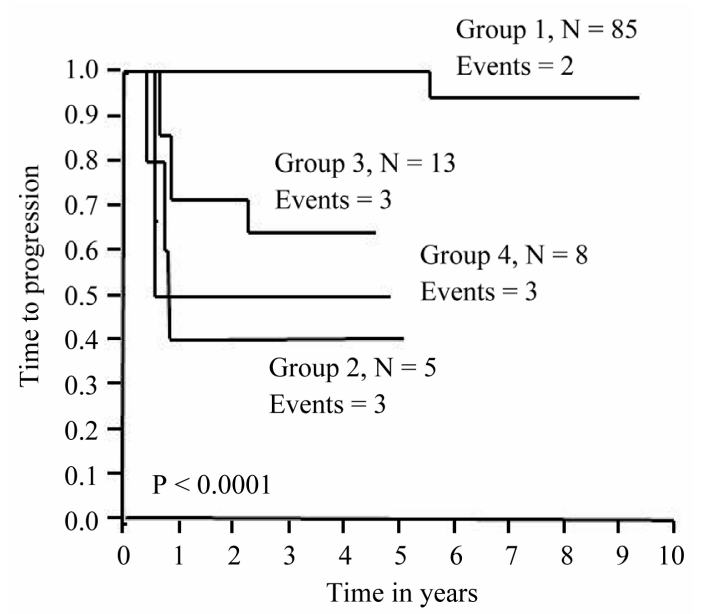

(b)

Figure 3. (a) Superior progression-free survival observed in patients in group 1 compared with the other groups; (b) Superior time to progression observed in patients in group 1 compared with the other groups. Group $1=$ negative interim positron emission tomography (PET)-scan and absolute lymphocyte/monocyte count ratio at diagnosis (ALC/ AMC-DX) $\geq 1.1$; group 2 = positive interim PET-scan and ALC/AMCDX $\geq 1.1$; group 3 = negative interim PET-scan and ALC/AMC-DX $<1.1$; and group $4=$ positive interim PET-scan and ALC/AMC-DX $<1.1$.

independent predictors for PFS and TTP, this finding led to investigate if there was any association between them. Patients with a negative int-PET presented with higher ALC/AMC-DX compared with patients with a positive int-PET. Furthermore, ninety percent of patients with an ALC/AMC-DX $\geq 1.1$ achieved a negative int-PET. The strong association between the int-PET and the ALC/ AMC-DX indirectly supports the crucial interaction between host immunity (i.e., ALC), tumor microenvironment (i.e., $\mathrm{AMC}$ ) and tumor burden (i.e., int-PET). To further clinically support the importance of the interaction between host immunity, tumor microenvironment and tumor burden using the int-PET and the ALC/AMC- 
DX, we categorized the patients into four groups based on positive versus negative int-PET and ALC/ AMC-DX $\geq 1.1$ versus ALC/AMC-DX $<1.1$. Patients in the group with negative int-PET (low tumor burden) and ALC/ AMC-DX $\geq 1.1$ (high host immunity and low immunosuppressive tumor microenvironment) experienced superior PFS and TTP in comparison with any other group with any negative marker of high tumor burden, low host immunity and/or immunosuppressive microenvironment.

A limitation of the IPS is that it only applies to cHL patients with advanced stage and not to limited stage cHL patients. Thus, we investigated if the ALC/AMCDX and the int-PET are prognostic factor for PFS and TTP according to the stage at diagnosis. We identified that both the ALC/AMC-DX and int-PET were predictors for PFS and TTP for cHL patients with limited stage and advanced stage. Furthermore, the prognosis for PFS and TTP for patients with a negative int-PET and an ALC/AMC-DX $\geq 1.1$ was superior to any group regardless of limited or advanced stage, suggesting that the combination of ALC/AMC-DX and int-PET can help to predict clinical outcomes in cHL patients by stage at presentation. Further studies are warrant to support this observation.

In contrast to Romano's article [4], the ALC/AMC-DX was found to be an independent of the int-PET to predict PFS and TTP. A difference between Romano's article and this study is that in Romano's study only $9 \%(11 / 115)$ cHL patients presented with an ALC/AMC-DX $<1.1$ and in our study it was $21 \%$, suggesting as Romano stated that in difference series, a different cut-off value should be calculated. This is well-documented by the paper of Koh et al. [3] where they used a cut-off of 2.9, instead of 1.1 to confirm that the ALC/AMC-DX was an independent predictor of survival in cHL. It is important to point out that even though statistical methods are useful to identify cut-off values of new prognostic bio-markers, new bio-markers need to be biologically sound to help us understand and improve clinical outcomes. This current study in addition to our previous publication [1], the studies by Koh [3] and Romano [4] continue to add more information to understand the role of the ALC/AMC-DX on survival in cHL.

To minimize the inherent biases of a retrospective study, the following steps were taken. With regards to selection bias, we included only patients with $\mathrm{cHL}$ and excluded patients with nodular lymphocyte predominant Hodgkin's lymphoma who are considered to have a different disease entity. We excluded patients treated upfront with palliative care or radiation alone, as chemotherapy and combination chemotherapy and radiation are considered the current standard of care for $\mathrm{cHL}$ patients. All patients were treated with the same chemotherapy regimen: ABVD. Patients who were positive for human immunodeficiency virus and concomitant autoimmune disease treated with immunosuppressive therapies were also excluded as these diseases and treatment directly influence ALC and AMC values. A strength of the study is the long-term follow-up of a well-defined group of patients with cHL. Secondly, the ALC/AMC-DX is simple, easily determined clinical biomarker that can be used to assess clinical outcomes in cHL in conjunction with the routine use of int-PET.

\section{Conclusion}

The combination of ALC/AMC-DX and int-PET as representative markers of host immunity, tumor microenvironment, and tumor burden can stratified clinical outcomes in cHL and further studies are warranted to confirm our findings.

\section{REFERENCES}

[1] L. F. Porrata, K. Ristow, J. P. Colgan, T. M. Habermann, T. E. Witzig, D. J. Inwards, et al., "Peripheral Blood Lymphocyte/Monocyte Ratio at Diagnosis and Survival in Classical Hodgkin's Lymphoma," Hematologica, Vol. 97, No 2, 2012, pp. 262-269. doi:10.3324/haematol.2011.050138

[2] Y. W. Koh, H. J. Kang, C. Park, D. H. Yoon, S. Kim, et al., "The Ratio of the Absolute Lymphocyte Count to the Absolute Monocyte Count Is Associated with Prognosis in Hodgkin's Lymphoma: Correlation with Tumor Associated Macrophages," Oncologist, Vol. 17, No. 6, 2012, pp. 871-880.

[3] T. Terasawa, J. Lau, S. Bardet, O. Couturier, T. Hotta, M. Hutchings, et al., "Fluorine-18-Fluorodeoxyglucose Positron Emission Tomography for Interim Response Assessment of Advanced-Stage Hodgkin's Lymphoma and Diffuse Large B-Cell Lymphoma: A Systemic Review," Journal of Clinical Oncology Vol. 27, No. 11, 2009, pp. 19061914. doi:10.1200/JCO.2008.16.0861

[4] A. Romano, C. Vetro, D. Donnarumma, S. Forte, M. Ippolito and F. Di Raimondo, "Early Interim 2-(1)Fluoro-2deoxy-D-glucose Positron Emission Tomography Is Prognostically Superior to Peripheral Blood Lymphocyte/Monocyte Ratio at Diagnosis in Classical Hodgkin's Lymphoma," Haematologica, Vol. 97, No. 6, 2012, pp. e22-e23. doi:10.3324/haematol.2012.064576

[5] C. J. Cox, T. M. Habermann, B. A. Payne, G. G. Klee, and R. V. Pierre. "Evaluation of the Coulter Counter Model S-Puls IV," American Journal of Clinical Pathology, Vol. 84, No. 3, 1985, pp. 297-306.

[6] D. Hasenclever, V. Diehl, J. O. Armitage, D. Assouline, M. Bjorkholm, E. Brusamolino, et al., "A Prognostic Score for Advanced Hodgkin's Disease," New England Journal of Medicine, Vol. 339, No. 21, 1998, pp. 15061514. doi:10.1056/NEJM199811193392104

[7] B. D. Chelson, B. Pfistner, M. E. Juweid, R. D. Gascoyne, 
L. Specht, S. J. Horning, et al., "Revised Response Criteria for Malignant Lymphoma," Journal of Clinical Oncology, Vol. 25, No. 5, 2007, pp. 579-586.

[8] E. Kaplan and P. Meier, "Nonparametric Estimation from Incomplete Observations," Journal of the American Sta- tistical Association Vol. 53, No. 282, 1958, pp. 457-481. doi: 10.1080/01621459.1958.10501452

[9] D. R. Cox, "Regression Models and Life-Tables," Journal of the Royal Statistical Society series B, Vol. 34, No. 2, 1972, pp. 187-202. 